\title{
A Review of the Current Evidence Connecting Seborrheic Dermatitis and Parkinson's Disease and the Potential Role of Oral Cannabinoids
}

\author{
Hope R. Rietcheck ${ }^{a} \quad$ Jalal Maghfour ${ }^{b}$ Chandler W. Rundle ${ }^{a}$
}

Sameeha S. Husayn ${ }^{c}$ Colby L. Presleyc Stefan H. Sillau ${ }^{d}$ Ying Liu $^{d}$

Maureen A. Leehey ${ }^{d}$ Cory A. Dunnick ${ }^{a}$ Robert P. Dellavalle ${ }^{a, e, f}$

a Department of Dermatology, School of Medicine, University of Colorado Anschutz Medical Campus, Aurora, CO, USA; ${ }^{b}$ Department of Medicine, Tulane University Medical School, New Orleans, LA, USA; ${ }^{c}$ College of Osteopathic Medicine, Rocky Vista University, Parker, CO, USA; ${ }^{d}$ Department of Neurology, School of Medicine, University of Colorado Anschutz Medical Campus, Aurora, CO, USA; ${ }^{e}$ Department of Epidemiology, Colorado School of Public

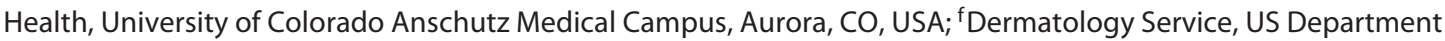
of Veterans Affairs Rocky Mountain Regional Medical Center, Aurora, CO, USA

\section{Keywords}

Parkinson's disease · Seborrheic dermatitis .

Neurocutaneous pathway · Cannabinoids · Cannabidiol

\begin{abstract}
Parkinson's disease (PD) is a neurodegenerative disorder associated with multiple comorbidities, including seborrheic dermatitis (SD), which develops in more than half of PD patients. SD in patients with PD can be severe and frequently intractable by traditional topical therapy. Cannabinoids possess anti-inflammatory and neuromodulatory properties working within the intrinsic endocannabinoid system, the activation of which may alleviate the motor symptoms of PD. The effect of cannabinoids on SD is unknown. Here we explore the pathophysiological mechanisms and possible therapeutic role of oral cannabinoids in PD patients with SD, and review speculative mechanisms underlying the association of PD and SD. Current data supporting the use of cannabinoids in both PD and SD, as well as oral cannabinoid
\end{abstract}

karger@karger.com

(c) 2020 S. Karger AG, Basel

www.karger.com/drm

Karger" safety and tolerability, are presented. Cannabinoids may provide the possibility of simultaneous treatment of both SD and PD. Specific SD studies and additional safety data on oral cannabinoids are needed.

(c) 2020 S. Karger AG, Basel

\section{Introduction}

Parkinson's disease (PD) is a progressive neurodegenerative disorder affecting 1-2\% of adults over the age of 65 years [1]. The pathophysiology of PD involves deterioration of dopaminergic neurons in the extrapyramidal tract of the midbrain. Motor symptoms, particularly akinesia, resting tremor, and rigidity, are a hallmark of the disease.

Premotor symptoms, such as constipation and changes in mood, have been recognized to precede classic PD presentation [2]. Several studies have also demonstrated Hope R. Rietcheck and Jalal Maghfour are co-first authors. 
an increased prevalence of skin diseases, such as seborrheic dermatitis (SD) and melanoma among PD patients [3]. In particular, SD occurs in as many as $50-59 \%$ of PD patients and is therefore considered to be a premotor feature of the disease $[4,5]$.

$\mathrm{SD}$ is a chronic, relapsing dermatitis characterized by erythema and scaly patches and has a predilection to symmetrically affect sebum-rich areas of the scalp, face, hairline, nasolabial folds, ears, and upper chest [6]. It is estimated to affect $1-3 \%$ of immunocompetent individuals and is more prevalent in men than women. SD is also characterized by a bimodal age distribution and is primarily seen in infants and adults over the age of 50 [6].

The most commonly recognized theory for the pathogenesis of SD is a nonspecific immune response to Malassezia yeast species [7]. Malassezia is a commensal organism that is present in lipid-rich and sebaceous skin areas. Development of SD is associated with the presence of Malassezia, ultraviolet radiation exposure, skin surface lipid composition, and overactivity of the sebaceous glands. In addition, it requires host susceptibility, e.g. immunocompromised individuals, as seen in organ transplants, human immunodeficiency virus infection, and cancer $[6,8]$. These observations suggest that defects in the immune system are important to the pathogenesis of the disease.

SD exerts a significant burden on patients due to its predilection to cosmetically apparent skin areas, which may lead to psychosocial distress with a negative impact on patients' quality of life [9]. The psychological distress has resulted in consumer incentive for disease treatment with an estimate of USD 1.2 billion spent for symptomatic relief. In fact, the total costs paralleled those associated with atopic dermatitis and pruritus at USD 339 million [10].

Although topical antifungals and corticosteroids are effective in mild-moderate SD, individuals with immune dysfunction, including PD, often develop severe forms of SD refractory to traditional therapy thus creating the need for systemic agents such as oral fluconazole and itraconazole $[8,11]$. However, these medications can be toxic and have variable levels of efficacy [8]. This indicates an unmet need of alternative therapy, ideally beneficial to patients with both PD and SD.

Neurocutaneous signaling is now recognized to be important in the pathogenesis of dermatological conditions such as atopic dermatitis and psoriasis [12]. In addition to their anti-inflammatory properties, cannabinoids are considered to have neuromodulatory properties and thus are able to target the neurocutaneous pathway [13]. A previous review has highlighted studies demonstrating the potential efficacy of cannabinoids in PD treatment [14]. Due to the increase in accessibility of cannabidiol (CBD) products on the market, a better understanding of the possible pathophysiological mechanisms and therapeutic role of oral cannabinoids in PD patients afflicted with SD is therefore of interest.

\section{Discussion}

\section{Parkinson's Disease and Its Relationship to SD}

The exact mechanism underlying the association of PD and SD is not fully clear. An increasing amount of evidence indicates direct involvement of Malassezia in the pathogenesis of PD $[5,15]$. This yeast species is also recognized as a factor in SD development, thereby constituting a common denominator in the pathogenesis of both SD and PD. Other possibilities for the strong association between SD and PD include gene polymorphisms GBA, LRRK2 and PINK1, which both play a role in lipid regulation and have been shown to confer an increased risk for PD development [16-18]. In particular, LRRK2 promotes formation of large lipid droplets while PINK1 increases their number and size [17, 18]. Additionally, a variant of the SNCA gene, which encodes for $\alpha$-synuclein, is dysregulated in PD and leads to the abnormal coating of lipid droplets, making them more susceptible to lipases [19]. This is of clinical importance as Malassezia is known to secrete lipases and it has been suggested that SNCA variants facilitate easier access to essential nutrients, allowing the fungus to infiltrate host tissue [19]. Further research is needed to elucidate Malassezia's potential direct contribution to the pathogenesis of both SD and $\mathrm{PD}$, but it may help explain the increased prevalence of SD among PD patients.

\section{Cannabinoids}

There are three classes of cannabinoids: phytocannabinoids (derived from plants), synthetic cannabinoids, and endogenous cannabinoids (also known as endocannabinoids) [20]. Tetrahydrocannabinol (THC) is one of the best-known types of phytocannabinoids. THC has been recognized for its psychoactive effects and is commonly used both recreationally and medicinally, with potential therapeutic effects in the treatment of PD [21].

$\mathrm{CBD}$ is a phytocannabinoid, which, in contrast to THC, has no psychoactive properties. It has been increasingly recognized for its potential efficacy in treating various inflammatory skin disorders. Although human stud- 
Fig. 1. Adapted from figures provided by Oláh et al. [37] and Tóth et al. [38], a simplified pathway of select CBD interactions with skin cells is illustrated. Downstream effects include maintenance and restoration of the skin barrier via reduction in inflammation and mediation of lipid production, as well as inhibition of sebaceous gland proliferation. CB1R, cannabinoid receptor; TRPV4, transient receptor potential vanilloid-4; NRP1, nuclear receptor protein 1 ; PPAR- $\gamma$, peroxisome proliferator-activated receptor $\gamma$.



ies in the use of CBD are limited, animal studies have demonstrated potential therapeutic benefits of CBD in treating atopic dermatitis and pruritus [22].

\section{The Role of Cannabinoid Use in the Treatment of PD}

The physiological role of the endocannabinoid system in modulating mood, behavior, and motor functions has been well documented in several literature reports [2325]. Endocannabinoid system dysregulation has been implicated in the pathogenesis of movement disorders including PD [26]. In experimental studies, it was shown that hypokinetic movements, as seen in $\mathrm{PD}$, are characterized by elevated endocannabinoid levels and a decrease in dopaminergic activity. The opposite effect was observed in hyperkinetic movements [27].

In one randomized control trial, the cannabinoid receptor (CB1R) agonist nabilone (oral CBD) was effective in the treatment of motor symptoms and improved dyskinesia secondary to levodopa [28]. Parallel to this, in a clinical study by Chagas et al. [29], CBD (300 mg/day) resulted in a significant improvement of quality of life measures and activity of daily life among PD patients without psychiatric comorbidities $(p<0.05)$. On the other hand, few clinical studies failed to demonstrate a significant difference in improvements of both motor and non-motor symptoms among PD patients [30]. It is im- portant to recognize, however, that there are limited studies investigating the efficacy of oral cannabinoids in PD treatment. Hence, it can be challenging to objectively conclude the efficacy of cannabinoids. Most recently, a clinical trial is investigating the safety and efficacy of CBD on tremor, motor symptoms, pain, depression, and additional symptoms in patients with PD [31].

\section{The Role of Cannabinoid Use in the Treatment of SD}

Considering Malassezia's implication in the pathogenesis of SD, mitigating fungal proliferation and localized inflammation is essential to treatment. Hence, topical antifungals (ketoconazole, ciclopirox) are often used to reduce the proliferation of Malassezia while topical corticosteroids are used to minimize skin inflammation. Additional level A recommended treatments include lithium (gluconate and succinate), which works by reducing the release of fatty acids in the skin, and tacrolimus [32]. For severe recalcitrant $\mathrm{SD}$, as seen in neurological disorders such as PD, only systemic antifungal therapies (itraconazole, terbinafine) have been shown to have some efficacy [33]. They are also known, however, to be hepatotoxic, and alternative therapies could be of benefit. Currently, both in vitro and in vivo reports support the potential use of cannabinoids in the treatment of inflammatory skin disorders although studies are few [34]. 
Lipid production by sebaceous glands is important in understanding the pathogenesis of SD. It has been shown that patients had more extensive skin desquamation than healthy individuals. Oleic acid has irritant and desquamative effects and is a byproduct of host lipid consumption by Malassezia [35]. Sebum dysregulation appears to play an important role in the pathogenesis of SD. Studying the effects of cannabinoids on sebum production may aid in understanding the potential benefits of oral cannabinoids in SD treatment.

Oral CBD and other non-psychoactive phytocannabinoid agents such as cannabichromene and tetrahydrocannabivarin use on human SZ95 sebocytes have resulted in a reduction in sebaceous gland proliferation and inhibition of lipid function [36]. This process appears to be mediated via the activation of transient receptor potential vanilloid-4. CBD was also shown to act through the nuclear receptor interacting protein 1 , which led to a decrease in lipid synthesis [37]. Furthermore, endocannabinoids are found within sebocytes and appear to mediate lipid synthesis and induce apoptosis via the CB2 pathway [38]. A simplified version of this mechanism is illustrated in Figure 1.

The activation of peroxisome proliferator-activated receptor $\gamma$ that results from cannabinoids binding to CB1 and $\mathrm{CB} 2$ receptors promotes not only the downregulation of proinflammatory cytokines, but also the upregulation of lipid production [39]. The latter was shown to be essential in restoring and maintaining the skin barrier. As discussed previously, disruption of the skin-lipid interface plays an important role in the pathogenesis of SD. Given the increased evidence on the role of the endocannabinoid system in regulating sebaceous gland function and lipid synthesis, its application to SD is promising.

Even though data is limited, both in vivo and in vitro studies have demonstrated that oral cannabinoids may be effective in treating a wide array of dermatological conditions as well as neurodegenerative disorders such as PD [40-43]. Patients with PD can present with severe SD and may benefit from a regimen that has the potential to target the endocannabinoid system found in both pilosebaceous units and the basal ganglia. Due to the increased burden of SD among PD patients, it may be of clinical utility to also assess the efficacy of CBD in SD treatment in these patients with a particular focus on symptoms of SD such as erythema, pruritus and scale, which can heavily impact a patient's quality of life.

\section{Safety and Tolerability of Cannabinoids}

While the use of oral cannabinoids is promising, limited safety profile data exist. However, recent studies have illustrated that $\mathrm{CBD}$ has a favorable safety profile. In one study, PD patients treated with oral CBD experienced an improvement in quality of life as assessed by the Parkinson's Disease Questionnaire PDQ-39, a questionnaire which assesses $\mathrm{PD}$-specific effects on dimensions of functioning and well-being; no adverse side effects were reported [29]. Currently, there is an ongoing phase 2 clinical trial assessing tolerability, safety and efficacy of oral cannabinoid in the treatment of dermatomyositis [43].

Due to variation in quality and processing of cannabinoid products, certain products may contain different active agents with various potencies such as synthetic cannabinoids, which have been shown to cause abdominal pain, hyperemesis syndrome, cannabinoid sensitization and/or allergy, and acute kidney injury [44]. While cannabinoids have antineoplastic effects, an in vivo study has demonstrated that tumor growth may occur during early stages following exposure to CBD [45]. Despite the conflicting data on potential adverse effects, it is important to understand the pharmacokinetics of cannabinoids and how drug-drug interaction can be of clinical concern.

There have been numerous reports on $\mathrm{CBD}$ and its effect on the CYP450 enzymes. Of most importance are isoforms CYP3A4 and 2CY19, as they are largely responsible for the biotransformation of CBD to its main and active metabolite [46]. When co-administered with a CYP3A4 inhibitor, such as oral ketoconazole, CBD bioavailability increases by $89 \%$ [47].

In the setting of severe, recalcitrant SD treated with known CYP inhibitors such as oral ketoconazole [48], clinicians should consider reducing CBD dosage to prevent any potential side effects. This is particularly important as ketoconazole is a known hepatotoxic agent and cannabinoids have been shown to result in an elevation of hepatic enzymes [46].

\section{Conclusion}

The intercommunication between the nervous system and the skin is now recognized to play a role in the pathogenesis of skin disorders and may shed light onto the link between SD and PD. Although the exact mechanism between the two entities is unclear, cannabinoids may be a promising therapy for the treatment of both $\mathrm{PD}$ and SD. 


\section{Key Message}

Seborrheic dermatitis (SD) is an inflammatory skin disorder that is more prevalent and severe in Parkinson's disease (PD) patients. Cannabinoids have the potential to treat both PD and SD.

\section{Conflict of Interest Statement}

Drs. Leehey and Liu are both investigators of an ongoing clinical trial assessing tolerability and efficacy of cannabidiol on motor symptoms in Parkinson's disease. The remaining co-authors have no conflicts of interest to disclose.

\section{Funding Sources}

No funding sources to disclose that are relevant to this review.

\section{Author Contributions}

H.R. and J.M. equally contributed to the work as primary cofirst authors by writing the original draft and performed extensive revisions. C.R. contributed in the writeup of the initial manuscript and provided revisions. S.H. and C.P. both aided in drafting the original manuscript and performed revisions. S.S., Y.L., and L.M. aided in generating the concept for the review and performed extensive revisions. C.D. provided guidance and aided in revisions of the paper. R.D., senior author, provided guidance to this project, performed extensive revisions, and aided with paper organization.

\section{References}

1 Schapira AH, Jenner P. Etiology and pathogenesis of Parkinson's disease. Mov Disord. 2011 May;26(6):1049-55.

2 Goldman JG, Postuma R. Premotor and nonmotor features of Parkinson's disease. Curr Opin Neurol. 2014 Aug;27(4):434-41.

3 Gregory R, Miller S. Parkinson's disease and the skin. Pract Neurol. 2015 Aug;15(4):246-9.

4 Antunes I, Purim KSM, Grande LL, Alberton NC, Navarro TFR, Winckler TCD. Dermatoses in parkinsonism: the importance of multidisciplinary follow-up. Rev Assoc Med Bras. 2019;65(6):791-795.

5 Arsic Arsenijevic VS, Milobratovic D, Barac AM, Vekic B, Marinkovic J, Kostic VS. A laboratory-based study on patients with Parkinson's disease and seborrheic dermatitis: the presence and density of Malassezia yeasts, their different species and enzymes production. BMC Dermatol. 2014 Mar;14(1):5.

6 Borda LJ, Wikramanayake TC. Seborrheic dermatitis and dandruff: A comprehensive review. J Clin Investig Dermatol. 2015 Dec; DOI: 10.13188a12373-11044.1000019.

7 Ashbee HR, Evans EG. Immunology of diseases associated with Malassezia species. Clin Microbiol Rev. 2002 Jan;15(1):21-57.

8 Dessinioti C, Katsambas A. Seborrheic dermatitis: etiology, risk factors, and treatments: facts and controversies. Clin Dermatol. 2013 Jul-Aug;31(4):343-51.

9 Karimkhani C, Dellavalle RP, Coffeng LE, Flohr C, Hay RJ, Langan SM, et al. Global Skin Disease Morbidity and Mortality: An Update From the Global Burden of Disease Study 2013. JAMA Dermatol. 2017 May;153(5): 406-12.

10 Seborrheic dermatitis by the numbers. American Academy of Dermatology Skin Disease Briefs 18-346, published 2017, updated 05/05/2018. Accessed 2020.

11 Ooi ET, Tidman MJ. Improving the management of seborrhoeic dermatitis. Practitioner. 2014;258(1768):23-26.
12 Elmariah SB. Adjunctive Management of Itch in Atopic Dermatitis. Dermatol Clin. 2017 Jul; 35(3):373-94.

13 Eagleston LR, Kalani NK, Patel RR, Flaten HK, Dunnick CA, Dellavalle RP. Cannabinoids in dermatology: a scoping review. Dermatol Online J. 2018 Jun;24(6):13030/qt7pn8c0sb.

14 More SV, Choi DK. Promising cannabinoidbased therapies for Parkinson's disease: motor symptoms to neuroprotection. Mol Neurodegener. 2015 Apr;10(1):17-17.

15 Laurence M, Benito-León J, Calon F. Malassezia and Parkinson's disease. Front Neurol. 2019 Jul;10:758.

16 Trinh J, Farrer M. Advances in the genetics of Parkinson disease. Nat Rev Neurol. 2013 Aug; 9(8):445-54.

17 Yu M, Arshad M, Wang W, Zhao D, Xu L, Zhou L. LRRK2 mediated Rab8a phosphorylation promotes lipid storage. Lipids Health Dis. $2018 \mathrm{Feb} ; 17(1): 34$

18 Narendra D, Walker JE, Youle R. Mitochondrial quality control mediated by PINK1 and Parkin: links to parkinsonism. Cold Spring Harb Perspect Biol. 2012;4(11):a011338.

19 Cole NB, Murphy DD, Grider T, Rueter S, Brasaemle D, Nussbaum RL. Lipid droplet binding and oligomerization properties of the Parkinson's disease protein alpha-synuclein. J Biol Chem. 2002 Feb;277(8):6344-52.

20 Khan MI, Sobocińska AA, Czarnecka AM, Król M, Botta B, Szczylik C. The Therapeutic Aspects of the Endocannabinoid System (ECS) for Cancer and their Development: From Nature to Laboratory. Curr Pharm Des. 2016;22(12):1756-66.

21 Kaur R, Ambwani SR, Singh S. Endocannabinoid System: A Multi-Facet Therapeutic Target. Curr Clin Pharmacol. 2016;11(2):110-7.

22 Hashim PW, Cohen JL, Pompei DT, Goldenberg G. Topical cannabinoids in dermatology. Cutis. 2017 Jul;100(1):50-2.

23 Fakhoury M. Role of the Endocannabinoid System in the Pathophysiology of Schizophrenia. Mol Neurobiol. 2017 Jan;54(1):768-78.
24 Vinod KY, Hungund BL. Role of the endocannabinoid system in depression and suicide. Trends Pharmacol Sci. 2006 Oct;27(10): 539-45.

25 Paloczi J, Varga ZV, Hasko G, Pacher P. Neuroprotection in Oxidative Stress-Related Neurodegenerative Diseases: Role of Endocannabinoid System Modulation. Antioxid Redox Signal. 2018 Jul;29(1):75-108.

26 Orgado JM, Fernández-Ruiz J, Romero J. The endocannabinoid system in neuropathological states. Int Rev Psychiatry. 2009 Apr;21(2): $172-80$.

27 Fernández-Ruiz J. The endocannabinoid system as a target for the treatment of motor dysfunction. Br J Pharmacol. 2009 Apr;156(7): 1029-40.

28 Sieradzan KA, Fox SH, Hill M, Dick JP, Crossman AR, Brotchie JM. Cannabinoids reduce levodopa-induced dyskinesia in Parkinson's disease: a pilot study. Neurology. $2001 \mathrm{Dec}$; 57(11):2108-11.

29 Chagas MH, Zuardi AW, Tumas V, PenaPereira MA, Sobreira ET, Bergamaschi MM, et al. Effects of cannabidiol in the treatment of patients with Parkinson's disease: an exploratory double-blind trial. J Psychopharmacol. 2014 Nov;28(11):1088-98.

30 Carroll CB, Bain PG, Teare L, Liu X, Joint C, Wroath $\mathrm{C}$, et al. Cannabis for dyskinesia in Parkinson disease: a randomized doubleblind crossover study. Neurology. 2004 Oct; 63(7):1245-50.

31 Leehey M. A study of tolerability and efficacy of cannabidiol on motor symptoms in Parkinson's disease. 2020. Available from: https:// ClinicalTrials.gov/show/NCT03582137. [cited 2020 Jul 6].

32 Mayser P, Schulz S. Precipitation of free fatty acids generated by Malassezia - a possible explanation for the positive effects of lithium succinate in seborrhoeic dermatitis. J Eur Acad Dermatol Venereol. 2016 Aug;30(8): 1384-9. 
33 Gupta AK, Bluhm R. Seborrheic dermatitis. J Eur Acad Dermatol Venereol. 2004 Jan;18(1): 13-26.

34 Das J, Majumdar M, Chakraborty U, Majumdar V, Mazumdar G, Nath J. Oral itraconazole for the treatment of severe seborrhoeic dermatitis. Indian J Dermatol. 2011 Sep-Oct; 56(5):515-6.

35 Tóth KF, Ádám D, Bíró T, Oláh A. Cannabinoid Signaling in the Skin: Therapeutic Potential of the "C(ut)annabinoid" System. Molecules. 2019 Mar;24(5):E918.

36 Thayikkannu AB, Kindo AJ, Veeraraghavan M. Malassezia-Can it be Ignored? Indian J Dermatol. 2015 Jul-Aug;60(4):332-9.

37 Oláh A, Tóth BI, Borbíró I, Sugawara K, Szöllõsi AG, Czifra G, et al. Cannabidiol exerts sebostatic and antiinflammatory effects on human sebocytes. J Clin Invest. 2014 Sep; 124(9):3713-24.

38 Tóth BI, Szallasi A, Bíró T. Transient receptor potential channels and itch: how deep should we scratch? Handb Exp Pharmacol. 2015;226: 89-133.
39 Dobrosi N, Tóth BI, Nagy G, Dózsa A, Géczy T, Nagy L, et al. Endocannabinoids enhance lipid synthesis and apoptosis of human sebocytes via cannabinoid receptor-2-mediated signaling. FASEB J. 2008 Oct;22(10):3685-95.

40 O'Sullivan SE. An update on PPAR activation by cannabinoids. Br J Pharmacol. 2016 Jun; 173(12):1899-910.

41 Spiera R, Hummers L, Chung L, et al. Safety and efficacy of lenabasum in a phase II, randomized, placebo-controlled trial in adults with systemic sclerosis. Arthritis Rheumatol. 2020 Aug;72(8):1350-60.

42 Burstein SH, Karst M, Schneider U, Zurier RB. Ajulemic acid: A novel cannabinoid produces analgesia without a "high". Life Sci. 2004 Aug;75(12):1513-22.

43 Robinson ES, Alves P, Bashir MM, Zeidi M, Feng R, Werth VP. Cannabinoid Reduces Inflammatory Cytokines, Tumor Necrosis Factor- $\alpha$, and Type I Interferons in Dermatomyositis In Vitro. J Invest Dermatol. 2017 Nov; 137(11):2445-7.

44 Werth VP, Hejazi E, Pena S, et al. Comparison of patients with dermatomyositis in a specialty clinic versus clinical trial with anabasum (JBT-101), a cannabinoid receptor type 2 agonist [abstract]. Arthritis Rheumatol. 2017; 68(suppl 10).
45 Cohen K, Weinstein AM. Synthetic and nonsynthetic cannabinoid drugs and their adverse effects - a review from public health perspective. Front Public Health. 2018 Jun;6:162.

46 Zheng D, Bode AM, Zhao Q, Cho YY, Zhu F, $\mathrm{Ma}$ WY, et al. The cannabinoid receptors are required for ultraviolet-induced inflammation and skin cancer development. Cancer Res. 2008 May;68(10):3992-8.

47 Brown JD, Winterstein AG. Potential Adverse Drug Events and Drug-Drug Interactions with Medical and Consumer Cannabidiol (CBD) Use. J Clin Med. 2019 Jul; 8(7):E989.

48 Stott C, White L, Wright S, Wilbraham D, Guy G. A Phase I, open-label, randomized, crossover study in three parallel groups to evaluate the effect of Rifampicin, Ketoconazole, and Omeprazole on the pharmacokinetics of THC/CBD oromucosal spray in healthy volunteers. Springerplus. 2013 May; 2(1):236 